Institute for Computational Mathematics

Hong Kong Baptist University

ICM Research Report

10-05 


\title{
Restoration of images corrupted by mixed Gaussian-Impulse noise via $l_{1}-l_{0}$ minimization
}

\author{
Yu Xiao ${ }^{\mathrm{a}}$, Tieyong Zeng ${ }^{\mathrm{b}, *}$, Jian $\mathrm{Yu}^{\mathrm{a}}$, Michael K. $\mathrm{Ng}^{\mathrm{b}}$ \\ ${ }^{a}$ School of Computer and Information Technology, Beijing Jiaotong University, \\ Beijing, China \\ ${ }^{b}$ Department of Mathematics, Hong Kong Baptist University, Kowloon Tong, Hong Kong
}

\begin{abstract}
In this paper, we study the restoration of images corrupted by Gaussian plus salt-and-pepper noise, and propose a $l_{1}-l_{0}$ minimization approach where the $l_{1}$ term is from impulse noise and the $l_{0}$ term is from the assumption that the clean image patches admit a sparse representation over certain unknown dictionary. The main algorithm contains three phases. First, we identify the outlier candidates which are likely to be corrupted by salt-and-pepper impulse noise. Second, we recover the image via dictionary learning on the free-outlier pixels. Finally, an alternating minimization algorithm is employed to solve the proposed minimization energy function, leading to an further restoration based on the recovered image in the second step. Comparative experiments are carried out to show the leading performance of the proposed method.
\end{abstract}

Keywords: Image restoration, Gaussian noise, Salt-and-Pepper noise, Dictionary learning

\section{Introduction}

Image restoration is an important task in the domain of image processing. The general idea is to estimate an ideal image $u$ from the observed noisy image $f$. In this paper, we restrict our attention to a mixed noise removal task. There are some different types of mixed noise models commonly considered

\footnotetext{
*Corresponding author

Email address: zeng@hkbu.edu.hk (Tieyong Zeng)
} 
in the literature, such as, blur and Gaussian (Impulse) noise[1, 2, 3, 4]; Poisson plus Gaussian noise[5] and Gaussian plus Impulse noise[6, 7, 8]. Here, we assume that the observed image $f$ is obtained from the last mixed noise model, which is defined as

$$
f=\mathbb{N}_{i m p}(u+b)
$$

where $b$ is an additive zero-mean Gaussian white noise with variance $\sigma^{2}$, and $\mathbb{N}_{i m p}$ denotes a process of image degradation with impulse noise. There are two common types of impulse noise: salt-and-pepper noise and randomvalued noise [9]. In this work, for simplicity, we only consider salt-and-pepper impulse noise. Denote $u_{i j}$ the gray value of an image $u$ at location $(i, j)$ and $\tilde{u}$ the noisy image. Suppose that the dynamic range of images is $\left[d_{\min }, d_{\max }\right]$, then the salt-and-pepper noise model is given as follows:

$$
\tilde{u}_{i j}=\left\{\begin{array}{cl}
d_{\min }, & \text { with probability } s / 2, \\
d_{\max }, & \text { with probability } s / 2, \\
u_{i j}, & \text { with probability } 1-s,
\end{array}\right.
$$

where $0 \leq s \leq 1$ is the noise density level of the salt-and-pepper noise. The gray values of corrupted pixels changes to either the maximum value $d_{\max }$ or the minimum value $d_{\text {min }}$.

In literature, most of denoising methods are aimed at removing either Gaussian or salt-and-pepper noise, which are much easier than the mixed noise removal. These two types of noise affect the image in totally different ways, leading to different denoising methods. For Gaussian noise removal, the commonly used methods include total-variation[10, 11, 12] and wavelet shrinkage approaches[13, 14]. The main drawback of the total-variation methods is that the texture information in images could be always oversmoothed [15]. Although the wavelet shrinkage methods perform much better for texture preserving, they may exhibit pseudo-Gibbs phenomena and bring artifacts in the recovered image[16]. Recently, Elad and Aharon introduced an effective denoising method via sparse and redundant representation over learned dictionaries, call K-SVD algorithm [17], which can preserve the details and texture efficiently. In [18], Dabov and coauthors proposed another sparse representation based denoising method in transform domain. We refer [19] for a comprehensive review on the developments of additive Gaussian noise removal methods.

For images corrupted by Impulse noise, one of the most popular methods is median filter for its denoising power and computational efficiency, see [20] 
and reference therein for some previous works about nonlinear digital filters. Recently, various modified median filters are proposed, e.g. the adaptive median filter[21], the multi-state median filter[22], the median filter based on homogeneity information[23, 24], convolution kernels based on filter[25]. The general idea of these filters is that the location of the candidate noisy pixels are detected and replaced by some values of the pixels in the corresponding neighbor windows, while all the other pixels unchanged. Although these filters gain a satisfactory results at the noise pixels detection, they can not preserve the original local feature since the noisy pixels are just simply replaced by some values computed according to other pixels in their neighborhood, e.g., the edges are not recovered satisfactorily, especially when the impulse noise level is high.

In order to better preserve the edge structures of images, various variational approaches have been proposed for Impulse noise removal [26, 27, $28,29,30]$. In [30], a $l_{1}$ data-fidelity term was introduced, which is much suitable for Impulse noise removal than $l_{2}$ norm used in $[26,27,28,29]$. This work provides an important improvement for Impulse noise removal. The main drawback of this method is that it changes the values of all the pixels in image, including the uncorrupted pixels, which should be fixed during the restoration process. In order to solve this problem, many effective two-phase methods are proposed $[31,32,3,33]$, combining various variational model with different median filters. The first phase of their methods is to detect the location of noisy pixels corrupted by impulse noise using median filters, and then employ an modified variational method to estimate the gray values for the noisy pixels in the second phase. Recently, some two-phase methods have been extended to process mixed noise. Cai et al proposed a modified two-phase method to deblur images corrupted by Impulse noise plus Gaussian noise[2]. In [4], a total variation based model was propose for Impulse and Gaussian noise removal. Lately, López-Rubio proposed a probabilistic theoretical framework to process images corrupted by Gaussian and uniform impulse noise[8]. Not limited to these methods for mixed noise removal, see $[6,7,1]$ for more information about image restoration under mixed noise.

All these methods referred above for impulse noise removal are pixel based method, considering each image pixel respectively, and so the local feature can not be preserved well, such as, texture structure, repeated patterns. It is surprising to notice that there are few work in literature handling impulse noise by the techniques of utilizing redundant prior in naturals images, which has been proved to be extremely successful for Gaussian noise removal. In 
this paper, inspired by the K-SVD and two-phase methods referred above, we propose a powerful patch based three-phase denoising method, combing the adaptive median filter[21] with an effective dictionary learning method: KSVD $[34,17]$, to recover images corrupted by Gaussian plus salt-and-pepper impulse noise. In the first stage, the impulse noise candidates are detected by the adaptive median filter, and then a modified K-SVD algorithm is used to recover the image, which is similar to the truncated K-SVD method in[35, 36]. Finally, we use an alternating minimization algorithm to solve the proposed denoising model. The main contributions of our method are clear: compared to classical impulse-related noise removal algorithm, we propose a doublesparsity approach which clearly outperforms previous works; compared to usual K-SVD, we consider an energy containing $l_{1}-l_{0}$ regularization where $l_{1}$ is proved more suitable for impulse noise removal.

The outline of the paper is as follows. Section 2 reviews some related works. In section 3 we propose the new image restoration method for Gaussian and salt-and-pepper noises removal. In section 4 , some numerical experiments are displayed to illustrate the performance of our method. The conclusions are presented in section 5 .

\section{Review of related works}

\subsection{Adaptive median filter}

Let $u_{i j}$ be the gray level of a noiseless image $u$ at pixel location $(i, j)$, and $\left[d_{\min }, d_{\max }\right]$ be the dynamic range of $u$. For salt-and-pepper impulse noise model, see formula (1), the gray level of the corrupted pixels are either $d_{\text {min }}$ or $d_{\max }$ and $f$ is the noisy image. Here we give a brief introduction of this filter. Firstly, we define a window $\mathrm{S}_{i, j}^{w}$ of size $w \times w$ centered at location $(i, j)$.

$$
\mathrm{S}_{i, j}^{w}=\{(k, l):|k-i| \leq w \text { and }|l-j| \leq w\}
$$

Let $w_{\max }$ be a pre-fixed value as the maximum window size. The main steps of the adaptive median filter (AMF) can be concluded as follows. For each pixel location $(i, j)$, do

1. Initial $w=3$.

2. Compute the minimum, median and maximum of the pixels values in $\mathrm{S}_{i, j}^{w}$ respectively: $d_{i, j}^{\min , w}, d_{i, j}^{\text {med,w }}$ and $d_{i, j}^{\max , w}$. 
3. If $d_{i, j}^{\min , w}<d_{i, j}^{\text {med,w }}<d_{i, j}^{\max , w}$, then go to last step. Otherwise enlarge the window size as $w=w+2$.

4. If $w<w_{\max }$ go to recompute the corresponding values in the new window as step 2. Otherwise replace $f_{i, j}$ by $d_{i, j}^{\text {med,w } w_{\max }}$.

5. If $d_{i, j}^{\min , w}<f_{i, j}<d_{i, j}^{\max , w}$, then $f_{i, j}$ is not a noise candidate, else we replace $f_{i, j}$ by $d_{i, j}^{m e d, w}$.

This adaptive median filter is good at detecting the impulse noise even at a high noise level when the window size is large enough. Adaptive median filter is robust in removing impulse noise while preserving sharpness. Since its simpleness and effective, many denoising methods use it to detect the corrupted pixels[31, 30, 2, 3, 4], also in our paper.

\section{2. $K-S V D$}

K-SVD is proposed by Aharon and Elad [34], which is used for additive Gaussian noise removal in [17]. Let us briefly review this method since it is main foundation of our denoising model for mixed noise. Suppose that a noisy image $f$ results from zero-mean Gaussian noise $b$ with a known standard deviation $\sigma$ superimposed on an original image $u$. The basic assumption of the K-SVD method is that each image patch (of fixed size) can be represented sparsely as a linear combination of atoms taken from a fixed dictionary. Using this assumption, the Gaussian noise removal task can be described as an energy minimization task:

$$
\left\{\hat{\alpha}_{i j}, \hat{D}, \hat{u}\right\}=\arg \min _{D, \alpha_{i j}, u} \lambda\|f-u\|_{2}^{2}+\sum_{i, j}\left\|D \alpha_{i j}-R_{i, j} u\right\|_{2}^{2}+\sum_{i, j} \mu_{i, j}\left\|\alpha_{i, j}\right\|_{0},
$$

where $R_{i, j} u$ extracts a patch of fixed size from the image in location $(i, j)$. The first term demands a proximity between the measured image, $f$, and its denoised unknown version $u$. The second term demands that each patch from the reconstructed image (denoted by $R_{i, j} u$ ) can be represented up to a bounded error by a dictionary $D$, with coefficients $\alpha_{i, j}$. The third part demands that the number of coefficients required to represent any patch is small. The values $\mu_{i, j}$ are patch-specific weights. The minimization of this functional with respect to its unknowns yields the denoising algorithm.

The choice of $D$ is of high importance to the performance of the algorithm. In $[34,17]$ it is shown that training can be done by minimization $(2)$. The 
Input: Noisy image $f$ with additive Gaussian noise.

Output: The reconstruction image $\hat{u}$.

Parameters: $\lambda$ (Lagrange multiplier); $C$ (noise gain); $J$ (number of iterations);

$K$ (number of dictionaries); $n$ (size of the patches);

$\sigma$ (standard deviation of Gaussian noise).

1. Initialization: set $u=f$, dictionary matrix $D^{(0)}=$ overcomplete DCT dictionary.

2. Repeat $J$ times

- Sparse Coding Stage: Use any pursuit algorithm to compute the representation vectors $\alpha_{i}$ for each example $x_{i}$ to minimize the function: $\forall_{i j} \min _{\alpha_{i j}}\left\|\alpha_{i j}\right\|_{0}$ s.t $\left\|R_{i j} u-D \alpha_{i j}\right\|_{2}^{2} \leq(C \sigma)^{2}$

- Dictionary Update Stage: For each column $l=1,2, \ldots, K$ in $D$.

- Select the patches $w_{l}$ that use this atom, $w_{l}=$ $\left\{[i, j] \mid \alpha_{i j}(l) \neq 0\right\}$

- For each patch $[i, j] \in w_{l}$, compute its residual without the contribution of the atom $\hat{d}_{l}$

$$
e_{i j}^{l}=R_{i j} u-D \alpha_{i j}+d_{l} \alpha_{i j}
$$

- Set $E_{l}=\left(e_{i j}^{l}\right)_{[i, j] \in w_{l}}$. Update $\hat{d}_{l}$ and the $\hat{\alpha}_{l}$ using SVD decomposition of $E_{l}$.

3. Reconstruction:

$$
u=\left(\lambda I+\sum_{i j} R_{i j}^{T} R_{i j}\right)^{-1}\left(\lambda f+\sum_{i j} R_{i j}^{T} D \alpha_{i j}\right)
$$

Table 1: K-SVD algorithm

proposed algorithm in $[34,17]$ is an iterative block-coordinate relaxation method which fixes all the unknowns except from the one to be updated.

Table 1 gives a description of K-SVD algorithm, which is a generalization of the K-means clustering algorithm. The setting of the parameters will be discussed in the experiments part. 


\section{Three-phase denoising approach}

Inspired by the two-phase method for impulse noise removal[3] and the $\mathrm{K}-\mathrm{SVD}[34,17]$ for dictionary learning, we proposed a $l_{1}-l_{0}$ minimization approach where the $l_{1}$ term is from impulse noise and the $l_{0}$ term is from the assumption that the clean image patches admit a sparse representation over certain unknown dictionary. A three-phases denoising algorithm is proposed to solve this $l_{1}-l_{0}$ minimization problem (3), and the main three steps can be concluded as follows.

- Identify the location of impulse noise (the outlier candidate pixels) using a median-type filter.

- Learn dictionaries from the free-outlier pixels and reconstruct the image via the learned dictionaries.

- Use an alternating minimization algorithm to solve the proposed $l_{1}-l_{0}$ denoising model, leading to an further restoration based on the recovered image in the second step.

Let $u$ be a clean image and $f$ its noisy version with Gaussian and saltand-pepper noise. The first step of our approach is to detect the outlier candidate pixels which are potentially corrupted by salt-and-pepper noise. Here, we use the adaptive median filter (AMF)[21] to detect salt-and-pepper noise, since it is simple and effective. Suppose $y \in R^{n \times n}$ is the filtered results by adaptive median filter. The candidates of noisy pixels contaminated by salt-and-pepper can be determined as follows:

$$
\mathcal{N}=\left\{(i, j) \in \mathcal{A}: y_{i j} \neq f_{i j} \text { and } f_{i j} \in\left\{d_{\min }, d_{\max }\right\}\right\}
$$

Accordingly, the left data samples are like to be uncorrupted which are defined as set $\mathcal{U}: \mathcal{U}=\mathcal{A} \backslash \mathcal{N}$.

Let $\mathcal{X}$ be the characteristic matrix of the set $\mathcal{U}$ defined as

$$
\mathcal{X}=\left\{\begin{array}{l}
1 \text { if }(i, j) \in \mathcal{U}, \\
0 \text { otherwise }
\end{array}\right.
$$

We are then interested in the following denoising model:

$$
\min _{u} \lambda\|\mathcal{X} \otimes(u-f)\|_{2}^{2}+\beta|(I-\mathcal{X}) \otimes(u-f)|+\sum_{i j}\left\|\left(D \alpha_{i j}-R_{i j} u\right)\right\|_{2}^{2}+\sum_{i, j} \mu_{i, j}\left\|\alpha_{i, j}\right\|_{0}
$$


where $\otimes$ is an enterwise multiplication between two matrices. The first term is a data-fidelity term, which considers the pixels that are probably not corrupted by salt-and-pepper noise, i.e only with Gaussian noise; the second data-fidelity term is an $\ell_{1}$ norm covering the outlier candidate pixels, since the outlier candidates are corrupted by salt-and-pepper impulse noise and $\ell_{1}$ is more reasonable than $\ell_{2}[30]$ for impulse noise. The last term is a sparsity representation for image patches via learned dictionaries. We use the above three phases to solve the proposed restoration model. After the first impulse noise detection step, the last two phases are explained respectively in the following two subsections.

\subsection{Restoration based on the free-outlier data}

After detection of the pixels corrupted by impulse noise, the left pixels in $\mathcal{U}$ are noisy but the noise is almost white Gaussian noise. Recently, for Gaussian noise model, Elad and Aharon have introduced a very effective denoising method via sparse and redundant representation over learned dictionaries, call K-SVD algorithm[17]. Here, we use K-SVD to learn dictionaries based on the pixels in $\mathcal{U}$, and then reconstruct the full noise image via a simple averaging between the patches' approximations and the noisy image. The modified energy function goes to formulation below:

$\hat{u}=\underset{u}{\arg \min }\|\mathcal{X} \otimes(u-f)\|_{2}^{2}+\sum_{i j} \mu_{i j}\left\|\alpha_{i j}\right\|_{0}+\sum_{i j}\left\|\left(R_{i j} \mathcal{X}\right) \otimes\left(D \alpha_{i j}-R_{i j} u\right)\right\|_{2}^{2}$.

The main steps of minimizing this modified K-SVD model stay as close as possible to the original K-SVD algorithm. More specifically, we aim at solving the energy minimization problem of (4) over the free-outlier candidate pixels. Integrating the location of the outlier candidate pixels requires the following key modifications to the basic algorithm:

- Sparse Coding: All projections in the OMP algorithm included only the free-outlier candidate pixels, and for this purpose, the dictionary elements were normalized so that the free-outlier indexes in each dictionary element have a unit norm. Given a fixed $D$,

$$
\hat{\alpha}_{i j}=\underset{\alpha}{\arg \min }\left\|\left(R_{i j} \mathcal{X}\right) \otimes\left(R_{i j} u-\hat{D} \alpha_{i j}\right)\right\|_{2}^{2}+\mu_{i j}\left\|\alpha_{i j}\right\|_{0}
$$

- Dictionary Update: Fix all $\alpha_{i j}$, and for each atom $d_{l}, l \in 1,2, \ldots, k$ in $D$. 
- Select the patches $w_{l}$ that use this atom, $w_{l}=\left\{[i, j] \mid \alpha_{i j}(l) \neq 0\right\}$

- For each patch $[i, j] \in w_{l}$, compute its residual

$$
e_{i j}^{l}=R_{i j} u-D \alpha_{i j}+d_{l} \alpha_{i j}
$$

and $\mathcal{X}_{i j}^{l}=R_{i j} \mathcal{X}$ is an index vector of the free-outlier candidate pixels in the small image patch of size $\sqrt{n} \times \sqrt{n}$ from location $[i, j]$ in the image.

- Set $E_{l}=\left(e_{i j}^{l}\right)_{[i, j] \in w_{l}}$ and $\mathcal{X}_{l}=\left(\mathcal{X}_{i j}^{l}\right)_{[i, j] \in w_{l}}$. Update $d_{l}$ by minimizing

$$
\hat{d}_{l}=\underset{\|d\|_{2}=1}{\arg \min }\left\|\mathcal{X}_{l} \otimes\left(E_{l}-d \alpha^{T}\right)\right\|_{2}^{2}
$$

For this optimal problem, we fix $\alpha$ and solve a simple quadratic term with respect to $d$.

- Reconstruction: Perform a weighted average

$$
u=\left(\lambda \mathcal{X} \otimes I+\sum_{i j} R_{i j}^{T} R_{i j}\right)^{-1}\left(\lambda \mathcal{X} \otimes f+\sum_{i j} R_{i j}^{T} \hat{D} \hat{\alpha_{i j}}\right)
$$

which is the solution of (4) with respect to $u$ when $D$ and $\alpha$ are assumed fixed. Note that the gray levels of outlier candidate pixels rely on the reconstruction from the optmal $D$ and $\alpha$, not relate to the noisy values corrupted by the impulse noise.

When the impulse noise level is low, the modified K-SVD (MK-SVD) can get satisfactory denoising results, however, when the impulse noise is high, the single MK-SVD can not learn good dictionaries from not enough free-outlier pixels. In order to improve the denoising results, we employ an alternating minimization algorithm to further restoration via the new dictionaries learned from the recovered image, leading to much more better dictionary atoms and better denoising results.

\subsection{Alternating minimization algorithm}

Since the learned dictionaries seriously rely on the trained image and the better dictionaries lead to better denoising results, a natural idea is that we use an alternating minimization algorithm to solve the proposed denoising model of (3): learn dictionaries from the recovered image and estimate a new 
Input: Noisy image $f$ corrupted by Gaussian plus Impulse noise.

Output: The reconstruction image $u$.

Parameters: $T$ (maximum number of iterations);

1. Detect the outlier candidates pixels with salt-and-pepper noise using AMF, and construct the characteristic matrix $\mathcal{X}$.

2. Compute the estimated image $\hat{u}$ by solving formulation (4).

3. Repeat $T$ times.

- Set the input image $f^{\prime}=\hat{u}$.

- Learn dictionary atoms $D$ and the corresponding coefficient matrix $\alpha$ using K-SVD, and the compute $W$ and $M$.

- Solve $\hat{u}$ as the minimization of formulation (5) via a fix-point iteration method.

4. Set the final recovered image $u=\hat{u}$.

Table 2: The full algorithm for Gaussian plus Impulse noise removal.

image via the new dictionaries. The experiments results show that iterated denoising via sparse representation gains high improvement compared to the single K-SVD for impulse noise removal, but which is not so powerful for Gaussian noise removal (gains much smaller improvement).

Compared with the original K-SVD algorithm, the sparse coding and dictionary updating steps remain unchanged, the main difference with the original K-SVD is the reconstruction step. In order to approximate the nonsmooth optimization problem (3), we introduce a weak smooth regularization as follows:

$$
\begin{aligned}
\mathcal{F}(u)=\lambda\|\mathcal{X} \otimes(u-f)\|_{2}^{2} & +2 \beta \sum_{(i, j) \in A} \sqrt{\overline{\mathcal{X}}_{i j}[u-f]_{i j}^{2}+\eta} \\
& +\sum_{i j}\left\|\left(D \alpha_{i j}-R_{i j} u\right)\right\|_{2}^{2}+\sum_{i j} \mu_{i j}\left\|\alpha_{i j}\right\|_{0},
\end{aligned}
$$

where $\eta \underset{\approx}{\approx}$. Then the gradient of $\mathcal{F}$ is given by:

$$
\nabla \mathcal{F}(u)=\lambda \mathcal{X} \otimes(u-f)+\beta(\overline{\mathcal{X}} \otimes(u-f))\left(\sqrt{[u-f]^{2}+\eta}\right)^{-1}+W u-M
$$


where $[\cdot]^{2}$ and $\div$ are entrywise square and division respectively, $\overline{\mathcal{X}}=I-\mathcal{X}$, $W=\sum_{i j} R_{i, j}^{T} R_{i j}$ and $M=\sum_{i j} R_{i, j}^{T} D \alpha_{i, j}$. Since $\mathcal{F}$ is convex, minimizing $\mathcal{F}$ is equivalent to solving $\nabla \mathcal{F}(u)=0$. Following [3], we minimize (5) by fixedpoint iteration method. Given $u^{t}$, we get $u^{t+1}$ by solving $u$ in the equation:

$$
\lambda \mathcal{X} \otimes(u-f)+\beta(\overline{\mathcal{X}} \otimes(u-f))\left(\sqrt{\left[u^{t}-f\right]^{2}+\eta}\right)^{-1}+W u-M=0 .
$$

Since $(7)$ is a linear equation and that has a closed-form solution of the form

$$
u^{t+1}=(\lambda \mathcal{X}+\beta \overline{\mathcal{X}} \otimes G+W)^{-1}(M+\lambda \mathcal{X} \otimes f+\beta \overline{\mathcal{X}} \otimes f \otimes G)
$$

where $G=\left(\sqrt{\left[u^{t}-f\right]^{2}+\eta}\right)^{-1}$.

\section{Experimental results}

In this section, experimental results are reported to validate the proposed method. We use eight test images of size $A \times B$ : Barbara $(512 \times 512)$, Boat $(512 \times 512)$, Lena $(512 \times 512)$, Hill $(512 \times 512)$, Pepper $(512 \times 512)$, Man $(512 \times 512)$, House $(256 \times 256)$ and Cameraman $(256 \times 256)$. Peak signal to noise ratio (PSNR) is used to measure the quality of the restored images which is defined as follows:

$$
P S N R=20 \log _{10} \frac{255}{\frac{1}{A B}\left\|u^{*}-u\right\|_{2}},
$$

where $\hat{u}$ and $u$ denote the restored image and the original image, respectively. In all experiments, we set the parameters of K-SVD as following: dictionary size: $64 \times 256$, image patch size: $8 \times 8, J=20$. We fix $\eta=1$, the window size $w_{\max }=19$ and $T=30$. The remaining parameter $\beta$ and $\lambda$ are chosen empirically such that it gives the best restoration measured in PSNR. In the simulations, images will be corrupted by Gaussian noise with standard deviation $\sigma$ and salt-and-pepper impulse noise with density level $s$. According to the value of $\sigma$, we divide the experiments into two parts: $\sigma=0$, i.e. only salt-and-pepper noise is considered; $\sigma \neq 0$, i.e. both Gaussian noise and salt-and-pepper noise are presented.

For $\sigma=0$, our algorithm is compared with six algorithms including AMF[21], Wang[25], Mila[30], Cai1[2], Cai2[3] and MK-SVD. Two typical 
Table 3: PSNR (dB) for various methods for Lena image at different noise density.

\begin{tabular}{ccccccccc}
\hline Noise density & Noisy & \multicolumn{2}{l}{ Method } \\
\cline { 3 - 8 } & & AMF & Wang & Mila & Cai1 & Cai2 & MK-SVD & Ours \\
\hline $10 \%$ & 15.12 & 37.24 & 40.35 & 43.28 & 42.86 & 43.79 & 46.31 & $\mathbf{4 6 . 5 4}$ \\
$20 \%$ & 12.19 & 35.07 & 36.58 & 39.91 & 39.33 & 40.45 & 42.19 & $\mathbf{4 3 . 0 1}$ \\
$30 \%$ & 10.37 & 32.71 & 33.64 & 37.15 & 37.00 & 37.74 & 39.14 & $\mathbf{4 0 . 2 6}$ \\
$40 \%$ & 9.14 & 30.98 & 31.81 & 35.36 & 35.38 & 35.93 & 36.27 & $\mathbf{3 7 . 2 3}$ \\
$50 \%$ & 8.15 & 29.22 & 30.19 & 33.39 & 33.73 & 33.88 & 33.84 & $\mathbf{3 5 . 2 7}$ \\
$60 \%$ & 7.35 & 27.43 & 28.92 & 31.46 & 32.21 & 32.31 & 31.53 & $\mathbf{3 3 . 0 6}$ \\
$70 \%$ & 6.70 & 25.75 & 27.75 & 29.47 & 30.53 & 30.32 & 29.40 & $\mathbf{3 0 . 7 5}$ \\
$80 \%$ & 6.13 & 23.88 & 26.25 & 27.70 & 28.29 & 28.28 & 27.35 & $\mathbf{2 8 . 4 0}$ \\
$90 \%$ & 5.60 & 21.04 & 24.13 & 24.41 & 25.13 & 25.29 & 25.07 & $\mathbf{2 5 . 7 0}$ \\
\hline
\end{tabular}

Table 4: PSNR (dB) for various methods for Barbara image at different noise density.

\begin{tabular}{ccccccccc}
\hline Noise density & Noisy & \multicolumn{2}{l}{ Method } \\
\cline { 3 - 8 } & & AMF & Wang & Mila & Cai1 & Cai2 & MK-SVD & Ours \\
\hline $10 \%$ & 15.23 & 28.75 & 33.34 & 35.12 & 35.14 & 35.11 & 44.20 & $\mathbf{4 4 . 3 5}$ \\
$20 \%$ & 12.21 & 27.66 & 29.88 & 31.67 & 31.82 & 31.58 & 39.46 & $\mathbf{4 0 . 2 9}$ \\
$30 \%$ & 10.49 & 26.41 & 27.69 & 29.51 & 29.67 & 29.36 & 36.23 & $\mathbf{3 7 . 3 9}$ \\
$40 \%$ & 9.28 & 25.22 & 26.07 & 27.93 & 28.17 & 27.76 & 33.42 & $\mathbf{3 4 . 4 0}$ \\
$50 \%$ & 8.28 & 24.13 & 24.74 & 26.65 & 26.84 & 26.60 & 30.99 & $\mathbf{3 1 . 9 0}$ \\
$60 \%$ & 7.50 & 22.97 & 23.53 & 25.48 & 25.74 & 25.51 & 28.87 & $\mathbf{2 9 . 7 0}$ \\
$70 \%$ & 6.81 & 22.62 & 21.91 & 24.30 & 24.85 & 24.58 & 26.82 & $\mathbf{2 7 . 6 2}$ \\
$80 \%$ & 6.23 & 20.66 & 21.56 & 22.70 & 23.81 & 23.67 & 24.73 & $\mathbf{2 5 . 3 2}$ \\
$90 \%$ & 5.72 & 18.93 & 20.37 & 19.34 & 22.64 & 22.52 & 22.60 & $\mathbf{2 2 . 9 0}$ \\
\hline
\end{tabular}


images are used to test these algorithms: Lena, the one with homogeneous region; Barbara, the one with much texture, and the salt and pepper noise levels are varied from $10 \%$ to $90 \%$ with increments of $10 \%$. The PSNR of the comparative methods with different noise levels are presented in Tables 3-4.

It is observed from Table 3 and 4 that the proposed method achieves higher PSNR than other noise reduction methods at every noise density. The PSNR of the proposed method is much high improvements over AMF, Wang, Mila, Cai1 and Cai2 when the noise density is relatively low $s \leq 60 \%$ for Lena and $s \leq 80 \%$ for Barbara. The performances of MK-SVD and our method are almost considerably at low density level $(s=10 \%)$, since the MKSVD can extract enough informative dictionary atoms from the incomplete images with few pixels are corrupted by impulse noise, leading to satisfactory denoising results as our method dose. However, when the noise density level is high, the dictionaries learned by MK-SVD can not preserve the local feature of the images while the proposed method can gain much better dictionaries by iteratively learning from recovered images, combining a $l_{1}$ norm for impulse noise removal, and so the PSNR of our method is roughly $1 \mathrm{~dB}$ improvement over MK-SVD at $20 \% \leq s \leq 80 \%$. For $s=90 \%$, almost $90 \%$ pixels are corrupted by impulse noise and either MK-SVD or our method can learn dictionaries containing enough local details of the ideal images through the left $10 \%$ noiseless pixels, but the PSNR values of our method are still little higher than all the other methods.

For $\sigma \neq 0$, we select three algorithms from the referred six methods to compare with the proposed algorithm, including Cai1[2] Cai2[3] and MKSVD, since the other three methods just consider the pixels contaminated by impulse noise and the pixels with Gaussian noise will not be recovered. Extensive experiments are conducted on all the eight test images. The salt and pepper noise levels are varied from 30\% to $70 \%$ with increments of $20 \%$, and Gaussian noise levels varies with $\sigma=5, \sigma=10$ and $\sigma=15$.

Fig. 1-4 show the denoising results for Lena and Barbara with Gaussian noise $\sigma=5$ and salt-and-pepper noise with noise levels $30 \%$ and $70 \%$. It can be seen that the proposed method outperforms the other three methods: better visual quality and higher PSNR values. In particular, a notable improvement is observed for Barbara. The texture areas of Barbara are well reconstructed since the image content structured objects (textures) can be well recovered by the learned dictionaries. Next,we demonstrate the learned dictionaries of Barbara and Lena with mixed noise $\sigma=5$ and $s=30 \%, 70 \%$. 


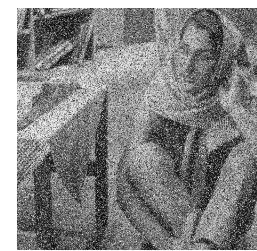

(a) 10.47

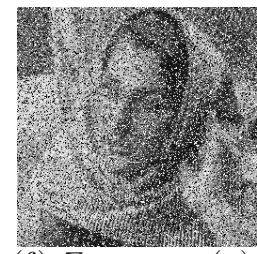

(f) Zoom on (a)

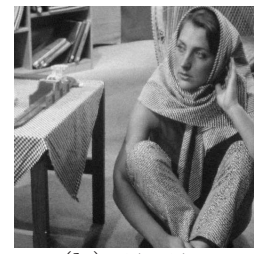

(b) 27.87

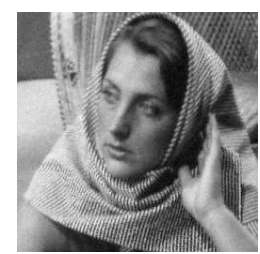

(g) Zoom on (b)

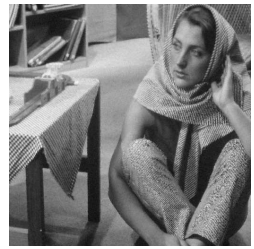

(c) 28.01

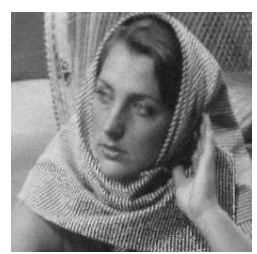

(h) Zoom on (c)

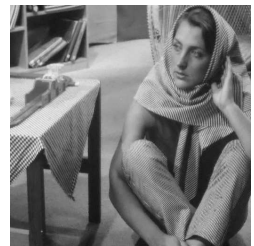

(d) 32.76

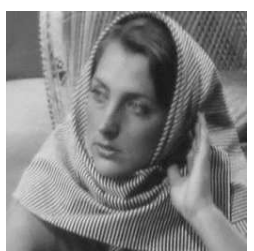

(i) Zoom on (d)

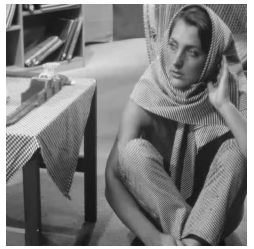

(e) 33.69

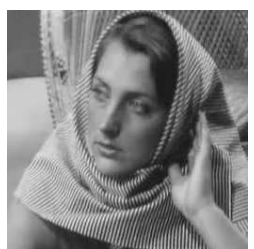

(j) Zoom on (e)

Figure 1: Denoising results of different methods on image Barbara corrupted by Gaussian noise and salt-and-pepper noise with $\sigma=5$ and $s=30 \%$ : $\operatorname{PSNR}(\mathrm{dB})$ values. From left to right, respectively, are presented: Noisy image,Cai1,Cai2, MK-SVD and Our algorithm.

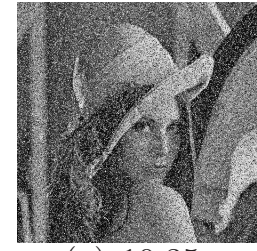

(a) 10.35

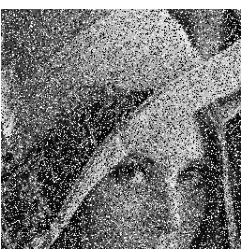

(f) Zoom on (a)

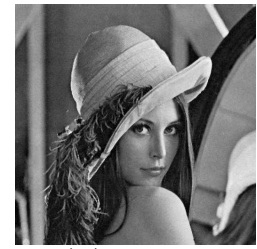

(b) 33.26

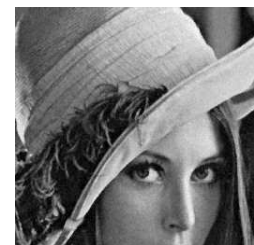

(g) Zoom on (b)

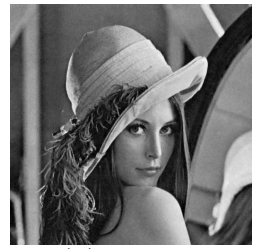

(c) 34.18

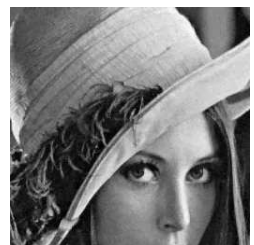

(h) Zoom on (c)

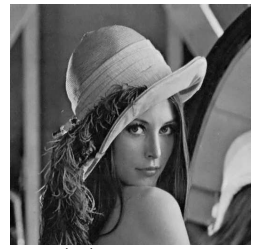

(d) 35.05

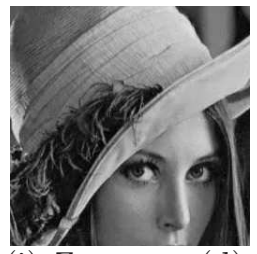

(i) Zoom on (d)

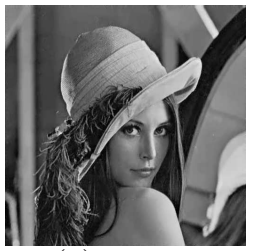

(e) 35.80

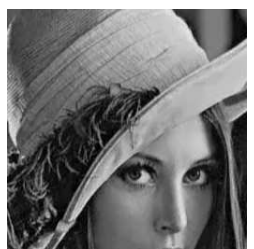

(j) Zoom on (e)

Figure 2: Denoising results of different methods on image Lena corrupted by Gaussian noise and salt-and-pepper noise with $\sigma=5$ and $s=30 \%: \operatorname{PSNR}(\mathrm{dB})$ values. From left to right, respectively, are presented: Noisy image,Cai1,Cai2, MK-SVD and Our algorithm.

Seen from Fig. 5 and Fig. 6, we can note that there are big differences between the two dictionaries learned from different images. Indeed, much more texture atoms are learned from Barbara. Moreover, the dictionaries learned by our method are much more clearer (smooth) and can describe the image feature much more effectively, especially under the impulse noise level 
$s=70 \%$, which leads to better denoising results.

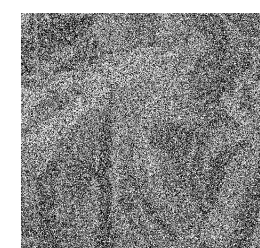

(a) 6.82

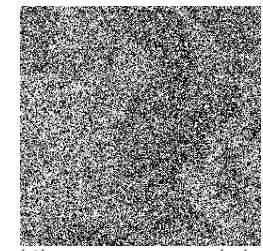

(f) Zoom on (a)

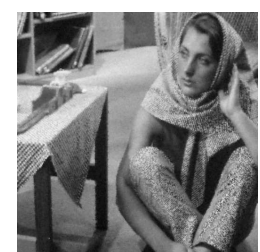

(b) 24.48

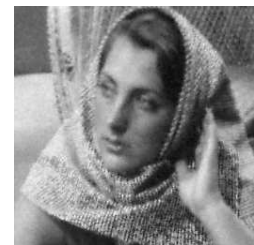

(g) Zoom on (b)

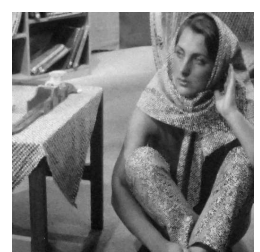

(c) 24.38

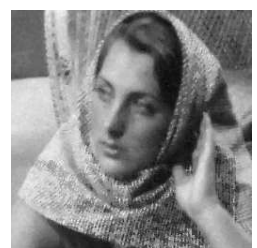

(h) Zoom on (c)

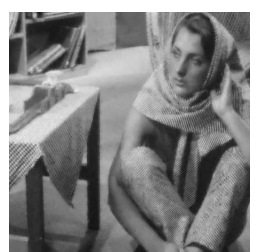

(d) 26.37

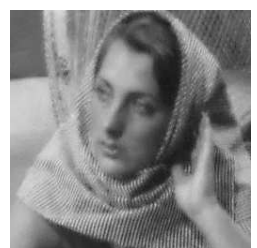

(i) Zoom on (d)

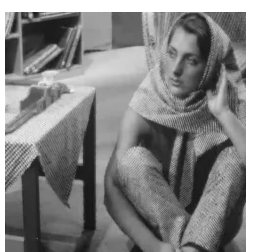

(e) 27.39

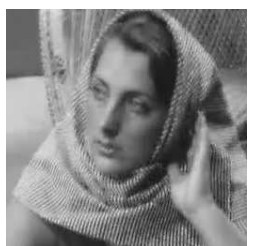

(j) Zoom on (e)

Figure 3: Denoising results of different methods on image Barbara corrupted by Gaussian noise and salt-and-pepper noise with $\sigma=5$ and $s=70 \%$ : PSNR(dB) values. From left to right, respectively, are presented: Noisy image, Cai1, Cai2, MK-SVD and Our algorithm.

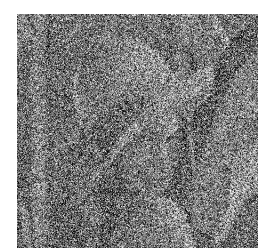

(a) 6.70

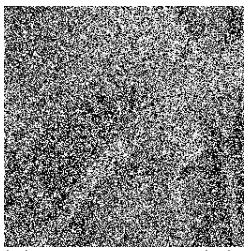

(f) Zoom on (a)

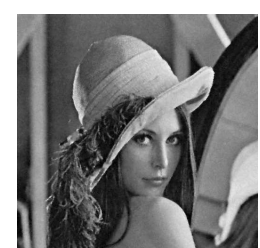

(b) 29.93

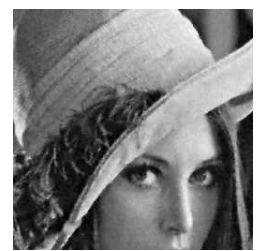

(g) Zoom on (b)

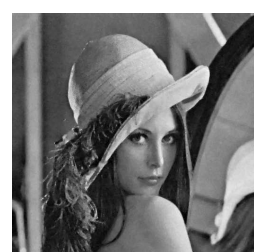

(c) 29.70

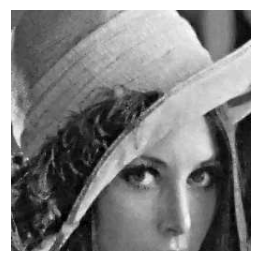

(h) Zoom on (c)

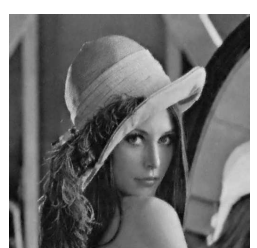

(d) 28.78

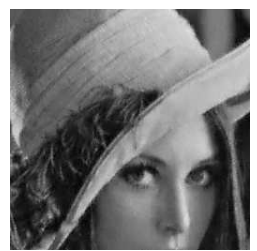

(i) Zoom on (d)

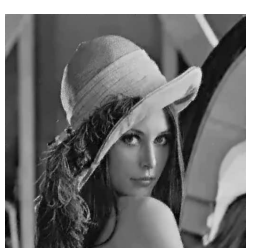

(e) 30.15

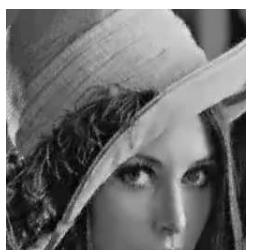

(j) Zoom on (e)

Figure 4: Denoising results of different methods on image Lena corrupted by Gaussian noise and salt-and-pepper noise with $\sigma=5$ and $s=70 \%$ : PSNR(dB) values. From left to right, respectively, are presented: Noisy image, Cai1, Cai2, MK-SVD and Our algorithm.

Table. 5 presents the quantitative results of the four denoising algorithms on all test images. Clearly, the proposed algorithm achieves promising denoising results: high improvement for images with much more texture areas, 


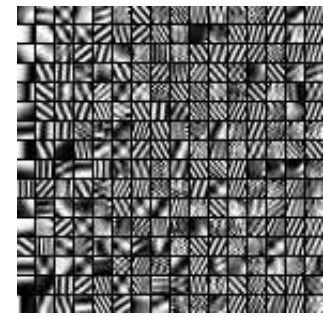

(a) MK-SVD, $30 \%$

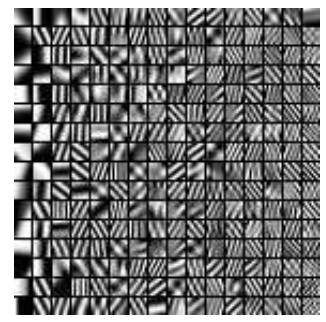

(b) Ours, $30 \%$

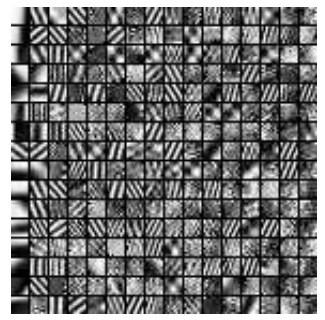

(c) MK-SVD,70\%

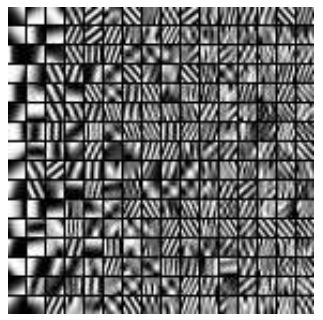

(d) Ours, $70 \%$

Figure 5: The learned dictionaries of Barbara under impulse noise with levels $30 \%, 70 \%$ and Gaussian noise with $\sigma=5$.

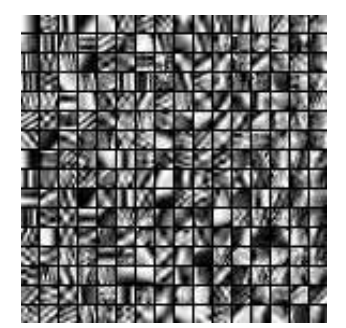

(a) MK-SVD, $30 \%$

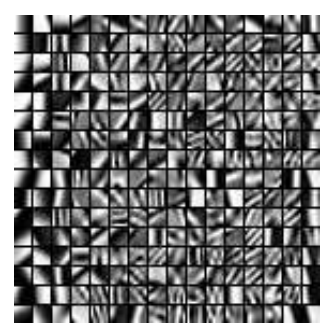

(b) Ours, $30 \%$

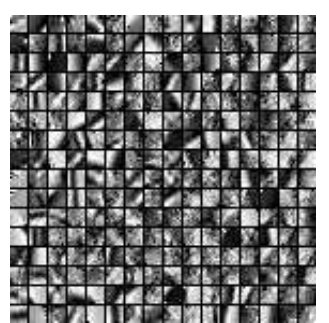

(c) MK-SVD, $70 \%$

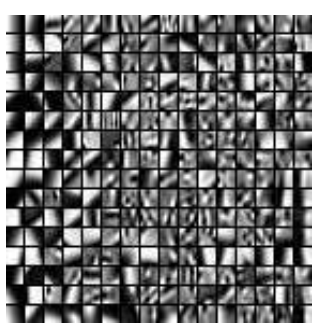

(d) Ours, $70 \%$

Figure 6: The learned dictionaries of Lena under impulse noise with levels 30\%,70\% and Gaussian noise with $\sigma=5$.

such as Barbara. The PSNR of our method is roughly $5 \mathrm{~dB}$ improvement over the Cai1 and Cai2 methods on Barbara when the impulse noise level is less than 70\%; and 1-2 dB improvement over the Cai1 and Cai2 algorithms on other test images except the two images: Cameraman and Man with $s=70 \%$, where Cai2 and our method perform considerably.

Comparing with Cai1 and Cai2, we see that the proposed method successfully suppresses the noise with the details and the texture of the images have been preserved very accurately. Comparing with MK-SVD, similar to the situation when $\sigma=0$, the MK-SVD can also get relatively satisfactory denoising results when the salt-and-pepper noise level $s=30 \%$ and Gaussian noise $\sigma \neq 0$ and the PSNR of our method is roughly $1 \mathrm{~dB}$ improvement over the MK-SVD when the salt-and-pepper noise level is high. 


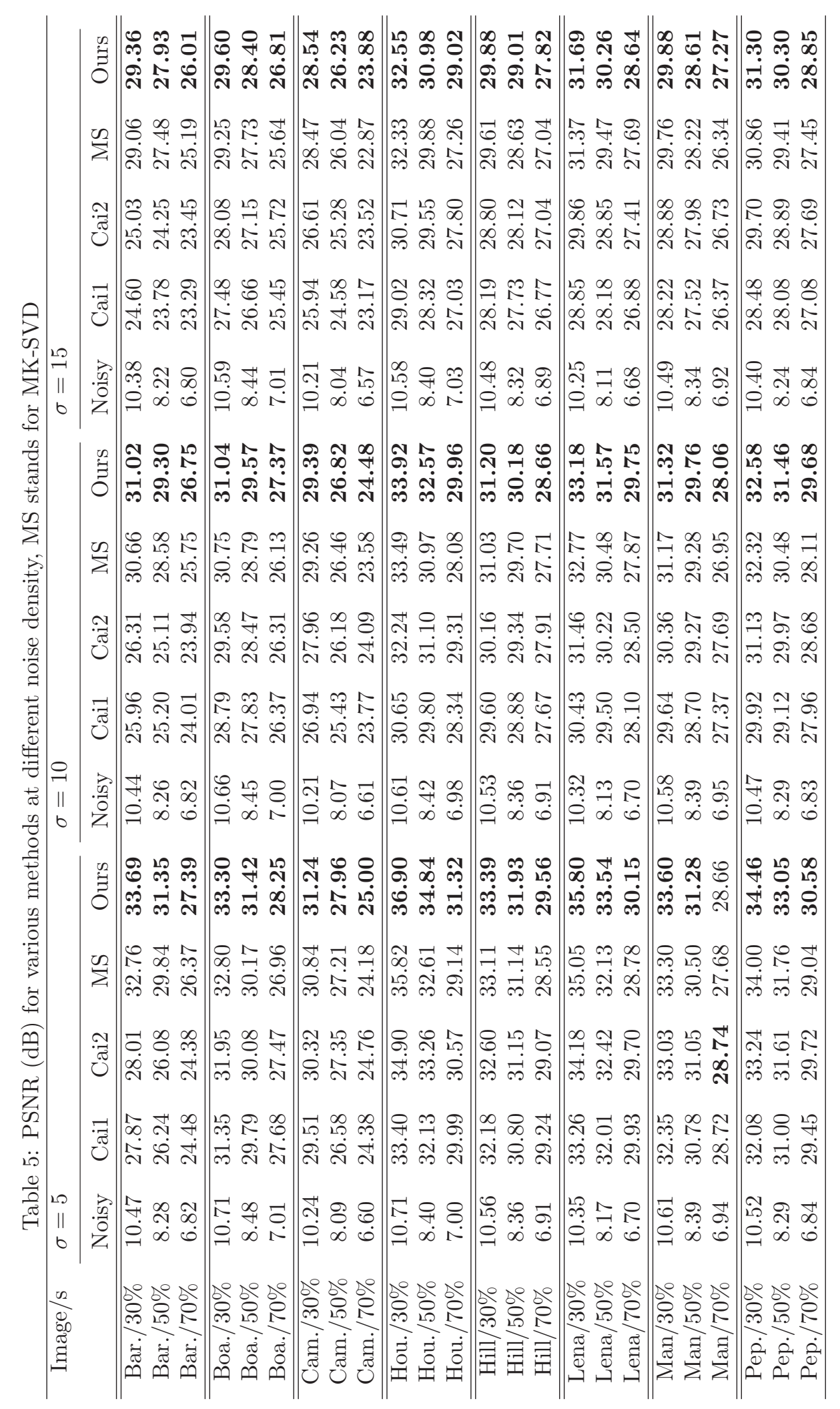




\section{Conclusion}

In this paper,we propose a powerful patch based three-phase denoising method to solve a $l_{1}-l_{0}$ denoising model. The proposed algorithm combines the adaptive median filter with an effective dictionary learning method: KSVD, to recover images corrupted by Gaussian plus salt-and-pepper impulse noise. The main contributions of our method are clear: compared to classical impulse-related noise removal algorithm, we propose a double-sparsity approach which clearly outperforms previous works; compared to usual KSVD, we consider an energy containing $l_{1}-l_{0}$ regularization where $l_{1}$ is proved more suitable for impulse noise removal.

The performance of the proposed method has been compared to some state of the art algorithms for both impulse noise and mixed noise removal tasks. The quantitative and qualitative results on test images demonstrate that our method can remove the noise efficiently while preserving the local feature of the image, even at a high impulse noise level $(\leq 70 \%)$ with Gaussian noise $\sigma \leq 15$.

One can further improve our results by using different noise detectors to improve the accuracy of the outlier detection especially when Gaussian noise density is high and other dictionary learned methods for high level impulse noise. Another possible extension is image deblurring with mixed noise, which is our future research topic.

\section{References}

[1] J. Liu, Z. Huan, H. Huang, An adaptive method for recovering image from mixed noisy data, International Journal of Computer Vision 85 (2009) 182-191.

[2] J.-F. Cai, R. Chan, M. Nikolova, Two-phase methods for deblurring images corrupted by impulse plus gaussian noise, Inverse Problem Imaging 2 (2008) 187-204.

[3] J.-F. Cai, R. Chan, M. Nikolova, Fast two-phase image deblurring under impulse noise, J Math Imaging Vison 36 (2010) 46-53.

[4] Y.-M. Huang, M. Ng, Y. Wen, Fast image restoration methods for impulse and gaussian noise removal, IEEE Transactions on Image Processing Letters 16 (2009) 457-460. 
[5] B. Zhang, M. Fadili, J. Starck, J. Olivo-Marin, Multiscale variancestabilizing transform for mixed-poisson-gaussian processes and its application in bioimaging, IEEE International Conference on Image Processing (2007) 233-236.

[6] S. Morillas, V. Gregori, A. Hervás, Fuzzy peer groups for reducing mixed gaussian-impulse noise from color images, IEEE Transactions on Image Processing 18 (2009) 1452-1466.

[7] J. Yang, H. Wu, Mixed gaussian and uniform impulse noise analysis using robust estimation for digital images, Proceedings of the 16th international conference on Digital Signal Processing (2009) 468-472.

[8] E. López-Rubio, Restoration of images corrupted by gaussian and uniform impulsive noise, Pattern Recognition 43 (2010) 1835-1846.

[9] H. Kong, L. Guan, A neural network adaptive filter for the removal of impulse noise in digital images, Neural Networks 9 (1996) 993-1003.

[10] L. Rudin, S. Osher, E. Fatemi, Nonlinear total variation based noise removal algortihm, Physica D. 60 (1992) 259-268.

[11] T. Chan, K. Chen, An optimization-based multilevel algorithm for total variation image denoising, SIAM J. Multiscale Modeling and Simulation. 5 (2006) 615-645.

[12] T. Chan, S. Esedoglu, F. Park, M. Yip, Recent developments in total variation image restoration, In Mathematical Models of Computer Vision (2005).

[13] M. Figueiredo, J. Bioucas-Dias, R. Nowak, Majorization-minimization algorithms for wavelet-base image restoration, IEEE Transactions on Image Processing 16 (2007) 2980-2991.

[14] F.Luisier, T. Blu, M. Unser, A new sure approach to image denoising: interscale orthonormal wavelet thresholding, IEEE Transactions on Image Processing 16 (2007) 593-606.

[15] A. Buades, B. Coll, J. Morel, A review of image denoising algorithms with a new one, Multiscale Model. Simul. 4 (2005) 490-530. 
[16] T. Chan, H. Zhou, Adaptive eno-wavelet transforms for discontinuous functions, In 12th International Conference on Domain Decomposition Methods (2001) 93-100.

[17] M. Elad, M. Aharon, Image denoising via sparse and redundant representations over learned dictionaries, IEEE Transactions on Image Processing 15 (2006) 3736-3745.

[18] K. Dabov, A. Foi, V. Katkovnik, K. Egiazarian, Image denoising by sparse 3d transform-domain collaborative filtering, IEEE Transactions on Image Processing 16 (2007) 3736-3745.

[19] V. Katkovnik, A. Foi, K. Egiazarian, J. Astola, From local kernel to nonlocal multiple-model image denoising, International Journal of Computer Vision 86 (2010) 1-32.

[20] J. Astola, P. Kuosmanen, Fundamentals of Nonlinear Digital Filtering, CRC, Boca Raton,United States of America, 1997.

[21] H. Hwang, R. Haddad, Adaptive median filters: new algorithms and results, IEEE Transactions on Image Processing 4 (1995) 499-502.

[22] T. Chen, H. R. Wu, Space variant median filters for the restoration of the impulse noise corrupted images, IEEE Transactions on Circuits and Systems 48 (2001) 784-789.

[23] G. Pok, J.-C. Liu, A. S. Nair, Selective removal of impulse noise based on homogeneity level information, IEEE Transactions on Image Processing 12 (2003) 85-92.

[24] H.-L. Eng, K.-K. Ma, Noise adaptive soft-switching median filter, IEEE Transactions on Image Processing 10 (2001) 242-251.

[25] S.-S. Wang, C.-H. Wu, A new impulse detection and filtering method for removal of wide range impulse noises, Pattern Recognition 42 (2009) 2194-2202.

[26] C. Bouman, K. Sauer, On discontinuity-adaptive smoothness priors in computer vision, IEEE Transactions on Pattern Analysis and Machine Intelligence 17 (1995) 579-586. 
[27] T. Chan, H. Zhou, R. Chan, A continuation method for total variation denoising problem, In Proceedings of SPIE Symposium on Advanced Signal Processing: Algorithm, Architecture, and Implementations 2563 (1995) 314-325.

[28] P. Charbonnier, L. Blanc-Féraud, G. Aubert, M. Barlaud, Deterministic edge-preserving regularization in computed imaging, IEEE Transactions on Image Processing 6 (1997) 298-311.

[29] C. Vogel, M. Oman, Fast, robust total variation-based reconstruction of noisy, blurred images, IEEE Transactions on Image Processing 7 (1998) 813-824.

[30] M. Nikolova, A variational approach to remove outliers and impulse nosie, Journal of Mathematical Imaging and Vision 20 (2004) 99-120.

[31] R. Chan, C. Ho, M. Nikolova, Salt-and-pepper noise removal by mediantype noise detector and edge-preserving regularization, IEEE Transactions on Image Processing 14 (2005) 1479-1485.

[32] R. Chan, C. Hu, M. Nikolova, An iterative procesure for removing random-valued impulse noise, IEEE Transactions on Signal Processing Letter 11 (2004) 921-924.

[33] S. Chen, X. Yang, G. Cao, Impulse noise suppression with an augmentation of ordered difference noise detector and an adaptive variational method, Pattern Recognition Letters 30 (2009) 460-467.

[34] M. Aharon, M. Elad, A. Bruckstein, The k-svd: An algorithm for designing of overcomplete dictionaries for sparse representation, IEEE Transactions on Image Processing 54 (2006) 4311-4322.

[35] J. Mairal, M. Elad, G. Sapiro, Sparse representation for color image restoration, IEEE Transactions on Image Processing 17 (2008) 53-69.

[36] J. Mairal, G. Sapiro, M. Elad, Learning multiscale sparse representations for image and videl restoration, SIAM Multiscale Modeling and Simulation 7 (2008) 214-241. 